\title{
Attosecond timing jitter pulse trains from semiconductor saturable absorber mode-locked Cr:LiSAF lasers
}

\author{
Duo Li, ${ }^{1, *}$ Umit Demirbas, ${ }^{1}$ Andrew Benedick, ${ }^{1}$ Alphan Sennaroglu, ${ }^{2}$ James G. Fujimoto, \\ and Franz X. Kärtner ${ }^{1,3}$ \\ ${ }^{1}$ Department of Electrical Engineering and Computer Science and Research Laboratory of Electronics, \\ Massachusetts Institute of Technology, Cambridge, MA 02139, USA \\ ${ }^{2}$ Laser Research Laboratory, Department of Physics, Koç University, Rumelifeneri, 34450 Istanbul, Turkey \\ ${ }^{3}$ Center for Free-Electron Laser Science, DESY and Department of Physics, University of Hamburg, D-22607 \\ Hamburg, Germany \\ "duoli@mit.edu
}

\begin{abstract}
The timing jitter of optical pulse trains from diode-pumped, semiconductor saturable absorber mode-locked femtosecond Cr:LiSAF lasers is characterized by a single-crystal balanced optical cross-correlator with an equivalent sensitivity in phase noise of $-235 \mathrm{dBc} / \mathrm{Hz}$. The RMS timing jitter is 30 attoseconds integrated from $10 \mathrm{kHz}$ to $50 \mathrm{MHz}$, the Nyquist frequency of the $100 \mathrm{MHz}$ repetition rate oscillator. The AM-toPM conversion induced excess phase noise is calculated and compared with experiment. The self-steepening effect is proven to be the dominant AM-toPM coupling mechanism, whereas the semiconductor saturable absorber operation does not adversely affect timing jitter. The results show that ultrafast Cr:LiSAF lasers are promising compact and efficient ultralow-jitter sources.
\end{abstract}

(C)2012 Optical Society of America

OCIS codes: (270.2500) Fluctuations, relaxations, and noise; (140.3480) Lasers, diodepumped; (140.4050) Mode-locked lasers; (140.5680) Rare earth and transition metal solid-state lasers.

\section{References and links}

1. J. Kim and F. X. Kärtner, "Attosecond-precision ultrafast photonics," Laser Photon. Rev. 4(3), 432-456 (2010).

2. J. A. Cox, J. Kim, and F. X. Kärtner, "Long-term, long-distance, all-optical synchronization of ultrafast fiber lasers at the quantum limit," 2010 Conference on Lasers and Electro-Optics (Cleo) and Quantum Electronics and Laser Science Conference (Qels) (2010).

3. J. Kim, F. Ludwig, M. Felber, and F. X. Kärtner, "Long-term stable microwave signal extraction from modelocked lasers," Opt. Express 15(14), 8951-8959 (2007).

4. J. Millo, R. Boudot, M. Lours, P. Y. Bourgeois, A. N. Luiten, Y. L. Coq, Y. Kersalé, and G. Santarelli, "Ultralow-noise microwave extraction from fiber-based optical frequency comb," Opt. Lett. 34(23), 3707-3709 (2009).

5. T. M. Fortier, M. S. Kirchner, F. Quinlan, J. Taylor, J. C. Bergquist, T. Rosenband, N. Lemke, A. Ludlow, Y. Jiang, C. W. Oates, and S. A. Diddams, "Generation of ultrastable microwaves via optical frequency division," Nat. Photonics 5(7), 425-429 (2011).

6. J. Kim, M. J. Park, M. H. Perrott, and F. X. Kärtner, "Photonic subsampling analog-to-digital conversion of microwave signals at 40-GHz with higher than 7-ENOB resolution," Opt. Express 16(21), 16509-16515 (2008).

7. H. A. Haus and A. Mecozzi, "Noise of Mode-Locked Lasers," IEEE J. Quantum Electron. 29(3), 983-996 (1993).

8. R. Paschotta, "Noise of mode-locked lasers (Part I): numerical model," Appl. Phys. B 79(2), 153-162 (2004).

9. R. Paschotta, "Noise of mode-locked lasers (Part II): timing jitter and other fluctuations," Appl. Phys. B 79(2), 163-173 (2004).

10. T. R. Schibli, J. Kim, O. Kuzucu, J. T. Gopinath, S. N. Tandon, G. S. Petrich, L. A. Kolodziejski, J. G. Fujimoto, E. P. Ippen, and F. X. Kaertner, "Attosecond active synchronization of passively mode-locked lasers by balanced cross correlation," Opt. Lett. 28(11), 947-949 (2003).

11. J. A. Cox, A. H. Nejadmalayeri, J. Kim, and F. X. Kärtner, "Complete characterization of quantum-limited timing jitter in passively mode-locked fiber lasers," Opt. Lett. 35(20), 3522-3524 (2010).

12. Y. Song, C. Kim, K. Jung, H. Kim, and J. Kim, "Timing jitter optimization of mode-locked Yb-fiber lasers toward the attosecond regime," Opt. Express 19(15), 14518-14525 (2011). 
13. U. Demirbas, A. Benedick, A. Sennaroglu, D. Li, J. Kim, J. G. Fujimoto, and F. X. Kärtner, "Attosecond resolution timing jitter characterization of diode pumped femtosecond Cr:LiSAF lasers," 2010 Conference on Lasers and Electro-Optics (Cleo) and Quantum Electronics and Laser Science Conference (Qels) (2010).

14. A. J. Benedick, J. G. Fujimoto, and F. X. Kärtner, “Optical flywheels with attosecond jitter,” Nat. Photonics 6(2), 97-100 (2012)

15. T. K. Kim, Y. Song, K. Jung, C. Kim, H. Kim, C. H. Nam, and J. Kim, "Sub-100-as timing jitter optical pulse trains from mode-locked Er-fiber lasers," Opt. Lett. 36(22), 4443-4445 (2011).

16. Y. Song, K. Jung, and J. Kim, "Impact of pulse dynamics on timing jitter in mode-locked fiber lasers," Opt. Lett. 36(10), 1761-1763 (2011)

17. R. Paschotta, "Timing jitter and phase noiseof mode-locked fiber lasers," Opt. Express 18(5), 5041-5054 (2010)

18. F. DeMartini, C. H. Townes, T. K. Gustafson, and P. L. Kelley, "Self-Steepening of Light Pulses," Phys. Rev. 164(2), 312-323 (1967).

19. H. A. Haus and E. P. Ippen, "Group velocity of solitons," Opt. Lett. 26(21), 1654-1656 (2001).

20. U. Demirbas, G. S. Petrich, D. Li, A. Sennaroglu, L. A. Kolodziejski, F. X. Kärtner, and J. G. Fujimoto, "Femtosecond tuning of Cr:colquiriite lasers with AlGaAs-based saturable Bragg reflectors," J. Opt. Soc. Am. B 28(5), 986-993 (2011).

21. U. Demirbas, D. Li, J. R. Birge, A. Sennaroglu, G. S. Petrich, L. A. Kolodziejski, F. X. Kaertner, and J. G. Fujimoto, "Low-cost, single-mode diode-pumped Cr:Colquiriite lasers," Opt. Express 17(16), 14374-14388 (2009).

22. D. Li, U. Demirbas, J. R. Birge, G. S. Petrich, L. A. Kolodziejski, A. Sennaroglu, F. X. Kärtner, and J. G. Fujimoto, "Diode-pumped passively mode-locked GHz femtosecond Cr:LiSAF laser with kW peak power," Opt. Lett. 35(9), 1446-1448 (2010).

23. S. Tsuda, W. H. Knox, S. T. Cundiff, W. Y. Jan, and J. E. Cunningham, "Mode-locking ultrafast solid-state lasers with saturable Bragg reflectors," IEEE J. Sel. Top. Quantum Electron. 2(3), 454-464 (1996).

24. U. Keller, K. J. Weingarten, F. X. Kärtner, D. Kopf, B. Braun, I. D. Jung, R. Fluck, C. Honninger, N. Matuschek, and J. Aus der Au, "Semiconductor saturable absorber mirrors (SESAM's) for femtosecond to nanosecond pulse generation in solid-state lasers," IEEE J. Sel. Top. Quantum Electron. 2(3), 435-453 (1996).

25. J. R. Birge and F. X. Kärtner, "Efficient optimization of multilayer coatings for ultrafast optics using analytic gradients of dispersion,” Appl. Opt. 46(14), 2656-2662 (2007).

26. "IEEE standard definitions of physical quantities for fundamental frequency and time metrology-random instabilities," IEEE STD 1139-2008, c1-35 (2009).

27. F. X. Kärtner, I. D. Jung, and U. Keller, "Soliton mode-locking with saturable absorbers,” IEEE J. Sel. Top. Quantum Electron. 2(3), 540-556 (1996).

28. J. P. Gordon and H. A. Haus, "Random walk of coherently amplified solitons in optical fiber transmission," Opt. Lett. 11(10), 665-667 (1986)

29. J. Kim, J. Chen, J. Cox, and F. X. Kärtner, “Attosecond-resolution timing jitter characterization of free-running mode-locked lasers," Opt. Lett. 32(24), 3519-3521 (2007).

30. T. R. Schibli, J. Kim, O. Kuzucu, J. T. Gopinath, S. N. Tandon, G. S. Petrich, L. A. Kolodziejski, J. G. Fujimoto, E. P. Ippen, and F. X. Kaertner, "Attosecond active synchronization of passively mode-locked lasers by balanced cross correlation," Opt. Lett. 28(11), 947-949 (2003).

31. SNLO nonlinear optics code available from A.V.Smith, AS-Photonics, Albuquerque, NM.

32. E. N. Ivanov, J. J. McFerran, S. A. Diddams, and L. Hollberg, "Noise properties of microwave signals synthesized with femtosecond lasers," IEEE Trans. Ultrason. Ferroelectr. Freq. Control 54(4), 736-745 (2007).

33. R. P. Scott, C. Langrock, and B. H. Kolner, "High-dynamic-range laser amplitude and phase noise measurement techniques,” IEEE J. Sel. Top. Quantum Electron. 7(4), 641-655 (2001).

34. K. Petermann, Laser Diode Modulation and Noise (Kluwer Academic Publishers, Norwell, MA, 1988).

35. L. Matos, O. D. Mücke, J. Chen, and F. X. Kärtner, "Carrier-envelope phase dynamics and noise analysis in octave-spanning Ti:sapphire lasers," Opt. Express 14(6), 2497-2511 (2006).

36. B. Sumpf, P. Adamiec, M. Zorn, H. Wenzel, and G. Erbert, "Nearly diffraction-limited tapered lasers at $675 \mathrm{~nm}$ with 1-W output power and conversion efficiencies above 30\%," IEEE Photon. Technol. Lett. 23(4), 266-268 (2011).

37. U. Demirbas, M. Schmalz, B. Sumpf, G. Erbert, G. S. Petrich, L. A. Kolodziejski, J. G. Fujimoto, F. X. Kärtner, and A. Leitenstorfer, "Femtosecond Cr:LiSAF and Cr:LiCAF lasers pumped by tapered diode lasers," Opt. Express 19(21), 20444-20461 (2011).

\section{Introduction}

Femtosecond mode-locked lasers have excellent noise properties. In particular, the extremely low timing jitter of optical pulse trains enables ultrafast lasers as ultralow jitter sources in numerous applications [1], including high precision synchronization of large-scale facilities [2], low phase noise microwave generation [3-5], high-speed, high-resolution optical sampling and analog-to-digital conversion [6]. The noise properties of mode-locked lasers have been studied analytically using soliton perturbation theory [7] and by numerical techniques $[8,9]$, which predict that the timing jitter of typical mode-locked solid-state lasers can easily reach the attosecond regime.

\#174914 - \$15.00 USD Received 24 Aug 2012; revised 18 Sep 2012; accepted 21 Sep 2012; published 27 Sep 2012

(C) 2012 OSA

8 October 2012 / Vol. 20, No. 21 / OPTICS EXPRESS 23423 
Balanced optical cross-correlation (BOC) measurement techniques [10] provide a direct optical domain method to measure timing jitter with high sensitivity and sufficient temporal detection range. This method has been utilized to completely characterize timing jitter up to the Nyquist frequency in passively mode-locked fiber lasers $[11,12]$ and solid-state lasers $[13,14]$. It is found that stretched-pulse fiber lasers operating at close-to-zero intracavity dispersion exhibit sub-100-as (from $10 \mathrm{kHz}$ to $38.8 \mathrm{MHz}$ ) quantum-limited timing jitter [15], and pulse dynamics and different mode-locked regimes influence the timing jitter power spectral density (PSD) [16, 17]. The lowest timing jitter reported to date is 13 as (from $100 \mathrm{~Hz}$ to $41.5 \mathrm{MHz}$ ) from Kerr-Lens Mode-locked (KLM) Ti:sapphire lasers [14]. In passively mode-locked solid-state lasers, however, excess phase noise due to AM-to-PM conversion has been observed in both Ti:sapphire and Cr:LiSAF lasers. It is therefore important to investigate the AM-to-PM mechanisms and to understand the impact of the mode-locking regimes on noise properties of pulse trains from ultrafast solid-state lasers. In particular, it is unclear whether temporal shifts of the pulses due to asymmetric pulse shaping by a slow saturable absorber significantly contribute to timing jitter. It has not been experimentally investigated if the operating point of the saturable absorber is critical with respect to timing jitter of optical pulse trains. Moreover, it is desirable to know to what extent the current timing jitter measurement on ultrafast solid-state lasers could be further improved, given that mode-locked fiber lasers have already reached the quantum limit at the sub-100-as level.

In this paper, we report on timing jitter measurements of semiconductor saturable absorber mode-locked femtosecond Cr:LiSAF lasers and discuss the impact of relative intensity noise (RIN) and operating point of the saturable absorber on timing jitter. By employing a singlecrystal BOC, we measure an RMS timing jitter of 30 as from $10 \mathrm{kHz}$ to $50 \mathrm{MHz}$ (Nyquist frequency). As will be shown in the next sections, saturable absorber mode-locking in Cr:LiSAF lasers does not adversely affect the timing jitter. The self-steepening effect $[18,19]$ is in fact the dominating factor resulting in AM-to-PM conversion of pump intensity noise, which is the same limitation as in KLM Ti:sapphire lasers. The 30-as RMS timing jitter is largely constrained by the timing jitter detection sensitivity. The paper is organized as follows. Section 2 introduces Cr:LiSAF lasers and summarizes quantum-limited timing jitter predicted by analytical modeling. Section 3 describes experimental details and results of timing jitter in-loop measurements with a single-crystal BOC. The results are further discussed in section 4, where laser intensity noise is characterized and the mechanisms for AM-to-PM conversion are discussed. Finally, Section 5 summarizes major findings and concludes with future work.

\section{Quantum-limited timing jitter of ultrafast Cr:LiSAF lasers}

Cr:Colquiriite lasers are low-cost, highly efficient and compact femtosecond pulse sources that are potential alternatives to Ti:sapphire lasers in some applications [20-22]. Because of their relatively low nonlinear refractive index, stable and self-starting continuous-wave modelocking can be initiated and sustained by semiconductor saturable absorbers [23, 24] and soliton pulse shaping.

The generic cavity layout of the Cr:LiSAF lasers used in this experiment is shown in Fig. 1. A standard astigmatically-compensated four-mirror cavity is used where two 637-nm, 220$\mathrm{mW}$ laser diodes (Opnext HL6385DG) pump the Brewster-cut Cr:LiSAF crystal (5.5 mm long, $1.5 \%$ doping). Cavity mirrors M1 and $\mathrm{M} 2$ have $75 \mathrm{~mm}$ radius of curvature (ROC), and mirror M3 (200 mm ROC) focuses the beam on the saturable absorber. Both laser diodes are temperature stabilized and driven by low-noise constant-current sources. In addition, each laser diode is driven at a certain combination of case temperature and injection current, under which condition high output power is obtained while mode hopping is not present. Intracavity group delay dispersion (GDD) is controlled by using specially designed dispersion compensating mirrors (DCMs) that have $-80 \pm 10 \mathrm{fs}^{2} \mathrm{GDD}$ from $800 \mathrm{~nm}$ to $950 \mathrm{~nm}$ [25]. The saturable absorber has $30 \mathrm{AlAs} / \mathrm{Al}_{0.15} \mathrm{Ga}_{0.85}$ As quarter-wave layers and a single $\mathrm{GaAs}$ quantum well buried in the top layer. The non-saturable loss is less than $0.5 \%$ at $850 \mathrm{~nm}$, and the modulation depth is $1 \%$. More details and other properties of the saturable absorber can be 
found in [23]. The 100-MHz laser generates sub-100 fs pulses centered around $860 \mathrm{~nm}$ with $\sim 1.2 \mathrm{~nJ}$ pulse energy.

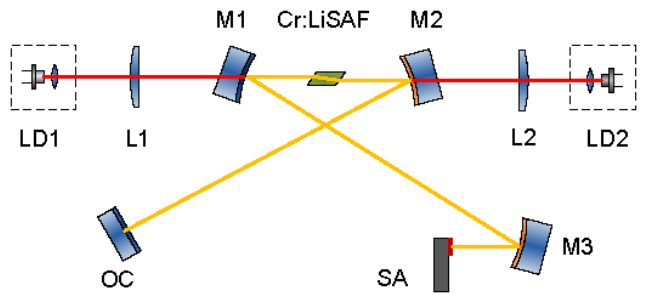

Fig. 1. Cavity layout of saturable absorber mode-locked Cr:LiSAF lasers. LD1, LD2: pump laser diodes. M1, M2, M3: cavity mirrors. L1, L2: input lenses. OC: output coupler. SA: saturable absorber

The timing jitter of pulse trains is the timing error $\Delta t$ of the power-weighted center of the pulse from the ideally equally-spaced temporal positions defined by the pulse repetition rate $f_{\text {rep }}$. Often, the timing jitter is represented as a phase error $\Delta \varphi=2 \pi \Delta t \cdot f_{\text {rep }}$ of the fundamental microwave signal derived upon detection of the pulse train. As a result, the phase representation $\Delta \varphi$ and its $\operatorname{PSD} S_{\phi}(f)$ scale with $f_{\text {rep }}$. In this paper, the pulse repetition rate is $f_{\text {rep }}=100 \mathrm{MHz}$. To convert the phase noise from the fundamental repetition rate to a 10 $\mathrm{GHz}$ carrier frequency, $S_{\phi}(f)$ is increased by $40 \mathrm{~dB}$. For better comparison with microwave technology, the phase noise is usually denoted by single-side band (SSB) phase noise PSD $\mathcal{L}(f)=S_{\phi}(f) / 2[26]$.

The sources of mode-locked laser timing jitter have been previously studied and summarized in Haus and Mecozzi's seminal paper [7]. Numerical simulations [8,9] confirm the Haus/Mecozzi analytical model and generalize it to pulse shapes other than the $\operatorname{sech}^{2}$ shape. In the case of a semiconductor saturable absorber mode-locked solid-state laser, the saturable absorber exerts impact on timing jitter of optical pulse trains. On one hand, a slow saturable absorber shapes pulses asymmetrically and therefore shifts the pulse center in every round-trip [27]. On the other hand, the saturable absorber's relatively narrow bandwidth limits the pulse's spectral width, limiting eventual Gordon-Haus jitter [28]. Consequently, the timing shift per round-trip $\Delta t$ is described by the following equation of motion derived with soliton perturbation theory [7]

$$
T_{\mathrm{R}} \frac{\partial}{\partial T} \Delta t=-2|D| \Delta p+T_{\mathrm{R}} S_{\mathrm{t}}(T)
$$

in which $T_{\mathrm{R}}$ is the round-trip time and equals $f_{\text {rep }}^{-1}$ for fundamentally mode-locked lasers. $T$ is time on the order of many round-trips, $D$ the intracavity dispersion, $\Delta p$ the center frequency shift per round-trip, and $S_{\mathrm{t}}(T)$ the direct noise source of timing fluctuation. The back coupling of continuum to solitary pulses is neglected. Moreover, it is assumed that the intracavity pulses are transform-limited, which is true for solitary pulses from saturable absorber modelocked Cr:LiSAF lasers.

Quantum-limited timing jitter assumes amplified spontaneous emission (ASE) as the sole noise source, which is approximately white noise with power per $\mathrm{Hz}$ $P_{\mathrm{ASE}}=\theta(2 g)(h v) / T_{\mathrm{R}}$, where $\theta$ is the excess noise factor, $\mathrm{g}$ the incremental field amplitude gain per round-trip, and $h v$ the photon energy. The ASE directly induces $1 /(2 \pi f)^{2}$-form random walk of pulses, resulting in a phase noise PSD [7]

\#174914 - \$15.00 USD Received 24 Aug 2012; revised 18 Sep 2012; accepted 21 Sep 2012; published 27 Sep 2012 (C) 2012 OSA 


$$
\begin{aligned}
\mathcal{L}(f) & =\frac{1}{2}\left(2 \pi f_{\text {rep }}\right)^{2} \frac{1}{(2 \pi f)^{2}}\left(\frac{\pi^{2} \tau^{2}}{6 w} P_{\mathrm{ASE}}\right) \\
& =\left(2 \pi f_{\text {rep }}\right)^{2} \frac{1}{12} \theta \frac{f_{\text {rep }}}{f^{2}} g \frac{\tau^{2}}{N_{0}}
\end{aligned}
$$

where $w$ is the intracavity pulse energy, $\tau=\tau_{\mathrm{FWHM}} / 1.76$ with $\tau_{\mathrm{FWHM}}$ being the full-width-halfmaximum (FWHM) intracavity pulse duration, and $N_{0}=w / h v$ the intracavity photon number.

In addition, the ASE-induced center frequency fluctuation $\Delta p$ also affects timing jitter according to Eq. (1). This is a major factor when the optical spectral width of the pulse only fills part of the gain bandwidth. Such frequency fluctuations couple to timing error and are damped with relaxation time $\tau_{\mathrm{p}}=3 T_{R} \Omega_{g}^{2} \tau^{2} / 4 g$, leading to a phase noise spectrum [7]

$$
\begin{aligned}
\mathcal{L}(f) & =\frac{1}{2}\left(2 \pi f_{\text {rep }}\right)^{2} \frac{4|D|^{2}}{T_{R}^{2}} \frac{1}{(2 \pi f)^{2}\left[(2 \pi f)^{2}+\tau_{\mathrm{p}}^{-2}\right]}\left(\frac{2}{3 w \tau^{2}} P_{\mathrm{ASE}}\right) \\
& =\left(2 \pi f_{\text {rep }}\right)^{2} \frac{3}{\pi^{2}} \theta\left(\frac{f_{\text {rep }}{ }^{3} \Omega_{g}{ }^{4}}{(2 g)^{2} f_{\text {rep }}{ }^{2}+(3 \pi)^{2} f^{2} \Omega_{g}{ }^{4} \tau^{4}}\right)|D|^{2} \frac{g}{f^{2}} \frac{\tau^{2}}{N_{o}}
\end{aligned}
$$

where $\Omega_{g}$ is the half-width-half-maximum (HWHM) gain bandwidth or cavity bandwidth, whichever is smaller. In the case of our Cr:LiSAF laser, the cavity bandwidth is limited by the saturable absorber, which supports femtosecond mode-locking with pulse center wavelengths from $\sim 850 \mathrm{~nm}$ to $\sim 870 \mathrm{~nm}$ which is experimentally measured in [20, 23], whereas a 100-fs pulse corresponds to only $\sim 8 \mathrm{~nm}$ bandwidth.

Upon detection of the laser pulses by a photodiode, shot noise of the pulse train indicates pulse position fluctuations, which leads to a white noise floor in timing jitter measurements given by [9]

$$
\mathcal{L}(f)=\left(2 \pi f_{\text {rep }}\right)^{2} 0.82 \frac{1}{f_{\text {rep }}} \frac{\tau^{2}}{N_{0}}
$$

This assumes that full intracavity power is received by a photodiode with ideal powerhandling capability. Experimentally accessible shot noise floor is higher, limited by the photodiode saturation and noise.

The dependence on $\tau^{2} / N_{0}$ of the results above clearly show that both short intracavity pulse duration and high intracavity photon number are important in order to achieve low timing jitter. Figure 2 illustrates the different contributions to quantum-limited timing jitter for a typical 100-MHz Cr:LiSAF laser. The Gordon-Haus jitter dominates for frequencies below $\sim 1 \mathrm{MHz}$, and the relaxation time $\tau_{\mathrm{p}} \approx 670 \mathrm{~ns}$ leads to a corner frequency at $\sim 237 \mathrm{kHz}$. At high frequencies, the shot-noise limited phase noise becomes dominant. This also implies that a measurement sensitivity better than $-280 \mathrm{dBc} / \mathrm{Hz}$ at $100-\mathrm{MHz}$ carrier is required to fully characterize the quantum-limited timing noise. The corresponding RMS timing jitter integrated from $10 \mathrm{kHz}$ to $50 \mathrm{MHz}$ is only $\sim 2$ as.

\section{Characterization of timing jitter in the optical domain}

Timing jitter is measured with a single-crystal BOC using two loosely-synchronized identical laser systems [29]. The system for timing jitter characterization is shown in Fig. 3(a). Laser beams from two $100-\mathrm{MHz} \mathrm{Cr}$ :LiSAF lasers are polarization combined by a polarizing beam 
splitter cube and sent to the BOC, which is shown in Fig. 3(b). The BOC is based on sum frequency generation (SFG) in a 400- $\mu$ m Beta Barium Borate (BBO) crystal cut for type-II phase matching at $830 \mathrm{~nm}$. The forward and backward SFG signal intensity is related to the temporal and spatial overlap of the two fundamental pulses. The signal from both passes is detected in an amplified balanced detector. To achieve low noise, high gain and large bandwidth, a Thorlabs PDB120A fixed-gain balanced detector was modified by for this experiment with two Hamamatsu S5712 photodiodes and a trans-impedance gain of $36 \mathrm{k} \Omega$ up to $\sim 20 \mathrm{MHz}$ bandwidth [14]. The noise floor is as low as $\sim 1 \mu \mathrm{V} / \sqrt{\mathrm{Hz}}$. The steering mirrors M3, M5 and M6 also serve as spectral filters that separate the SFG signal from the fundamental input signal. The output of the balanced detector is split by a 20:1 voltage divider. The low voltage output is connected to a servo controller (New Focus LB1005), which drives the PZT in laser 2 and synchronizes the cavity length to that of laser 1 . The high voltage output is sent to an RF spectrum analyzer (Advantest R3267) and an oscilloscope. The measured power contains equal contributions from the two uncorrelated pulse trains. The corresponding voltage PSD is therefore divided by 2, and finally converted to SSB phase noise at $100-\mathrm{MHz}$ carrier frequency using the measured phase error discriminator slope.

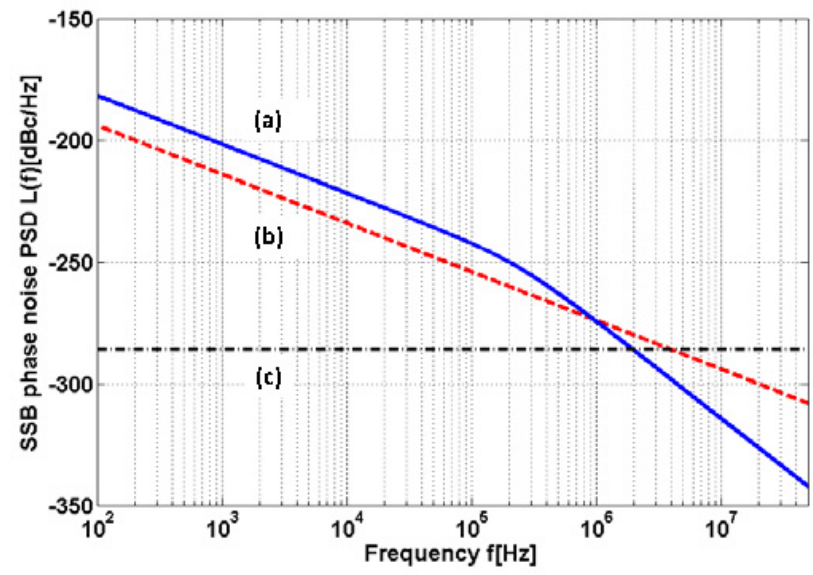

Fig. 2. Quantum-limited SSB phase noise at $100-\mathrm{MHz}$ carrier. (a) Gordon-Haus jitter with HWHM gain bandwidth of $25.5 \mathrm{THz}(850 \mathrm{~nm}-870 \mathrm{~nm})$. (b) Phase noise only due to direct phase noise from ASE. (c) Phase noise due to shot noise. Other parameters used: $\theta=1$, intracavity power $=6 \mathrm{~W}, 2 \mathrm{~g}=0.03, \mathrm{D}=-100 \mathrm{fs} 2$ and pulse width $=80 \mathrm{fs}$.

The phase noise measurement sensitivity is determined by the timing discriminator, which can be understood by analyzing the cross-correlation of both passes. For simplicity, assume that the two lasers generate identical pulses with $1 / \mathrm{e}$ pulse duration $\tau_{0}$ and peak intensity $I$. If only temporal overlap is considered, for $\operatorname{sech}^{2}$-shaped pulses, the balanced detector output voltage is

$$
\begin{aligned}
V(\tau) \propto & \tau_{0} G L^{2} I^{2} \csc h^{2}\left(\frac{t_{1}-t_{2}+\tau}{\tau_{0}}\right)\left[\left(\frac{t_{1}-t_{2}+\tau}{\tau_{0}}\right) \cot h\left(\frac{t_{1}-t_{2}+\tau}{\tau_{0}}\right)-1\right] \\
& -\tau_{0} G L^{2} I^{2} \csc h^{2}\left(\frac{2 t_{1}-2 t_{2}+\tau}{\tau_{0}}\right)\left[\left(\frac{2 t_{1}-2 t_{2}+\tau}{\tau_{0}}\right) \cot h\left(\frac{2 t_{1}-2 t_{2}+\tau}{\tau_{0}}\right)-1\right]
\end{aligned}
$$

where $G$ is the trans-impedance gain, $L$ is the crystal length, and $t_{1}-t_{2}=\left(v_{g 1, e}^{-1}-v_{g 2, o}^{-1}\right) L / 2$ with $v_{g 1, e}$ and $v_{g 2, o}$ the group velocities of the two fundamental pulses with extraordinary and ordinary axes of the crystal. Figure 4(a) shows the calculated SFG signals from the forward 
pass, backward pass and balanced signal according to Eq. (5), assuming a 400- $\mu \mathrm{m}$ BBO crystal and 100-fs pulses. The linear range around the zero crossing of the solid curve is used as a time-to-voltage discriminator to measure the instantaneous time delay of the fundamental pulse pair. Equation (5) can be used to optimize the discriminator slope by fine-tuning group delay and therefore the separation of forward and backward cross-correlation curves. In a single-crystal BOC, the group delay is often introduced by birefringence of the nonlinear crystal. In a two-crystal setup, however, additional dispersive material can be employed to generate desired group delay maximizing the slope [30]. The 400- $\mu \mathrm{mBBO}$ crystal generates $\sim 72$ fs group delay, whereas the optimum value for 100 -fs pulses is $\sim 120 \mathrm{fs}$, which corresponds to $\sim 2 \mathrm{~dB}$ enhancement of discriminator slope. Moreover, when the BOC is used to synchronize the pulse repetition rates of the two lasers, Eq. (5) tells the relative delay between the pulse pair when a phase-lock loop (PLL) locks the balanced signal to zero.

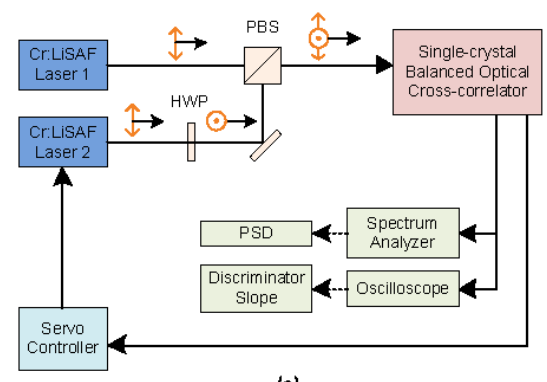

(a)

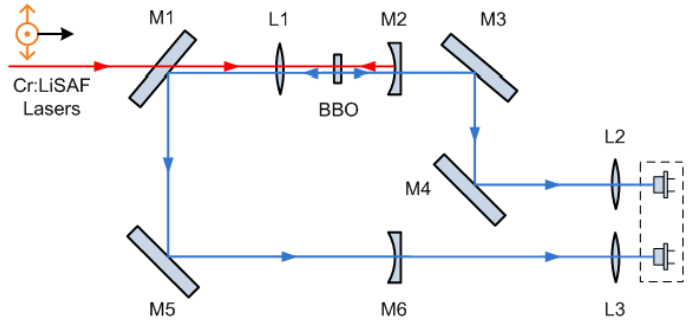

(b)

Fig. 3. Schematic of measurement system. (a) System block diagram. HWP: half-wave plate. PBS: polarizing beam-splitter. (b) BOC using a type-II phase-matched BBO crystal.

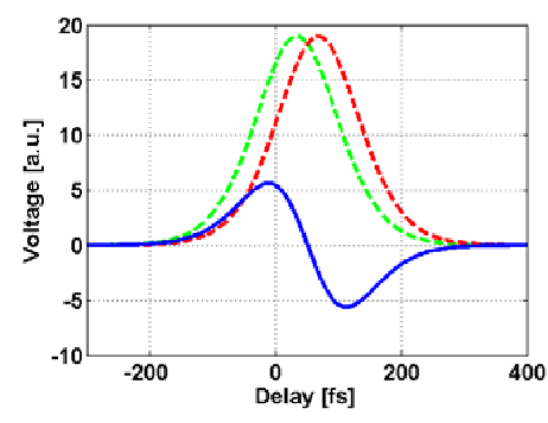

(a)

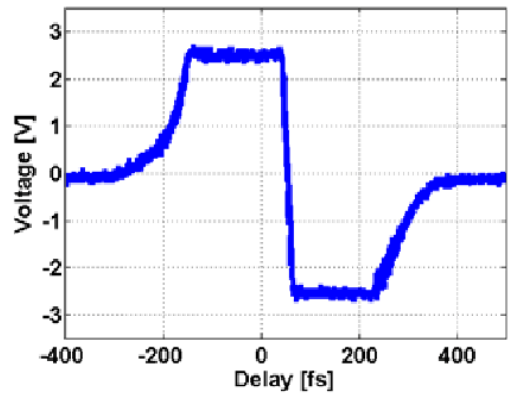

(b)

Fig. 4. Timing discriminator. (a) Calculated cross-correlation signals (dashed) and balanced output (solid) using Eq. (5). (b) Measured phase error discriminator, with a zero-crossing slope of $240 \mathrm{mV} / \mathrm{fs}$.

Experimentally, the balanced cross correlator characteristic is measured with an oscilloscope when the repetition rate of one laser is slightly detuned relative to the other, and the slope is calculated by differentiating the linear range. A typical voltage output is shown in Fig. 4(b). The zero-crossing of the balanced signal corresponds to a relative delay of $51 \mathrm{fs}$ between the fundamental pulses. The optimum SFG efficiency for the current experimental configuration is estimated to be $3 \times 10^{-3}$ using the software SNLO [29], whereas the measured efficiency is $100 \mu \mathrm{W} / 110 \mathrm{~mW} \approx 10^{-3}$, which is close to the theoretical efficiency.

In contrast to a conventional two-oscillator phase noise measurement using direct photodetection, the BOC method is immune to AM-to-PM conversion in the photo detection process. The excess phase noise usually originates from amplitude fluctuations of the input signal [32]. In the case of the BOC, intensity noise is suppressed by strong common-mode 
rejection of the balanced detector. Although both timing error and intensity fluctuations of input pulses result in a change of SFG intensity, the intensity fluctuations in forward and backward passes are identical and therefore are eliminated in the differential output. Ideally, this assumes an infinite common-mode rejection ratio (CMRR) and balanced optical power on both photodiodes. Real CMRR is both finite and frequency-dependent.

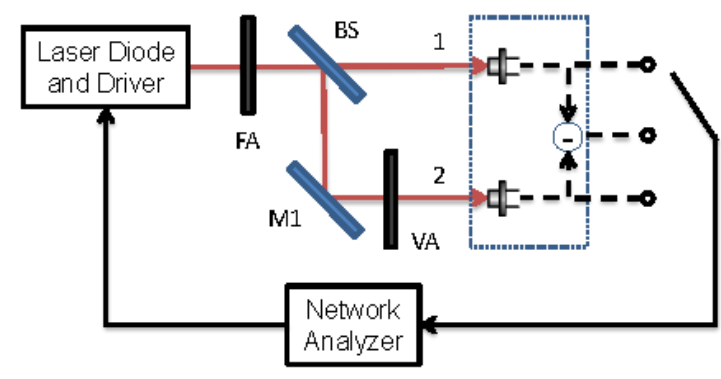

(a)

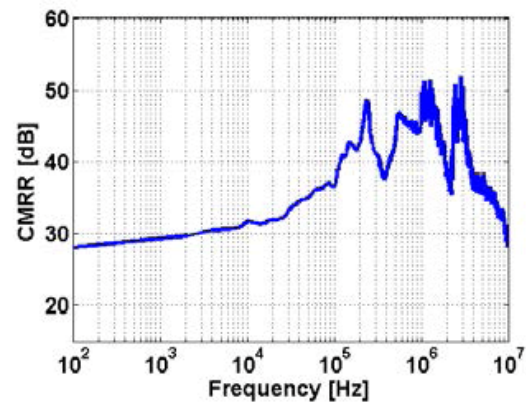

(b)

Fig. 5. CMRR of the modified balanced detector. (a) Measurement setup. FA: fixed attenuator; BS: beam splitter; M1: metallic mirror; VA: variable attenuator (b) CMRR when the two channels are balanced.

The CMRR of the modified balanced detector is characterized with the setup shown in Fig. 5(a). A 661-nm laser diode is driven by a low-noise diode driver (ILX Lightwave ILX3620) that has a modulation bandwidth of $1 \mathrm{MHz}$. A fixed attenuator controls the input optical power on the photodiodes. The attenuated beam is then split into two paths, in one of which a continuously variable attenuator is used to balance the two channels or to introduce certain mismatch. The network analyzer (Agilent E4395A) coherently measures the response of either an individual photodiode or the balanced output at various modulation frequencies. The laser diode is modulated from $100 \mathrm{~Hz}$ to $1 \mathrm{MHz}$ with the driver's internal modulation circuit, and from $1 \mathrm{MHz}$ to $10 \mathrm{MHz}$ with a bias-T. The real CMRR is then the difference of differential-mode gain and common-mode gain in logarithmic scale. The response of the diode driver and laser diode is cancelled out in the subtraction. In any measurement, the network analyzer response is calibrated to a thru. In general, the CMRR is greater than $28 \mathrm{~dB}$ within the frequency range of interest. It is also measured that with a small $10 \%$ power mismatch on the photodiodes, the CMRR degrades to 10-15 dB. This emphasizes the importance to balance the incident power on both photodiodes.

Figure 6 summarizes timing jitter measurement expressed as SSB phase noise at $100-\mathrm{MHz}$ carrier frequency. The low-frequency phase noise is suppressed by the feedback loop. The detection sensitivity is $-235 \mathrm{dBc} / \mathrm{Hz}$, limited by the discriminator slope and the noise floor of the balanced detector. The integrated timing jitter from $10 \mathrm{kHz}$ to $50 \mathrm{MHz}$, the Nyquist frequency, is 30 as, most of which results from the detection noise floor, which is already reached beyond $100 \mathrm{kHz}$.

The measurement accuracy is determined by that of the RF spectrum analyzer and the discriminator slope. Most spectrum analyzers measure power within a certain resolution bandwidth (RBW), and the PSD is derived using $\mathrm{dBm} / \mathrm{Hz}=\mathrm{dBm}-10 \lg (\alpha \cdot R B W)$, where

$\alpha$ is a factor that corrects for system error and filter shape. The value of $\alpha$ can be determined using the built-in noise measurement function of the instrument, which displays a calibrated noise PSD within a given frequency range. The system error of PSD measurements in this experiment is $\sim 1 \mathrm{~dB}$. In addition, the relative error of sensitivity $(\mathrm{mV} / \mathrm{fs})$ is experimentally determined to be less than $20 \%$. In total, the above system errors result in an uncertainty $< \pm$ 10as. 


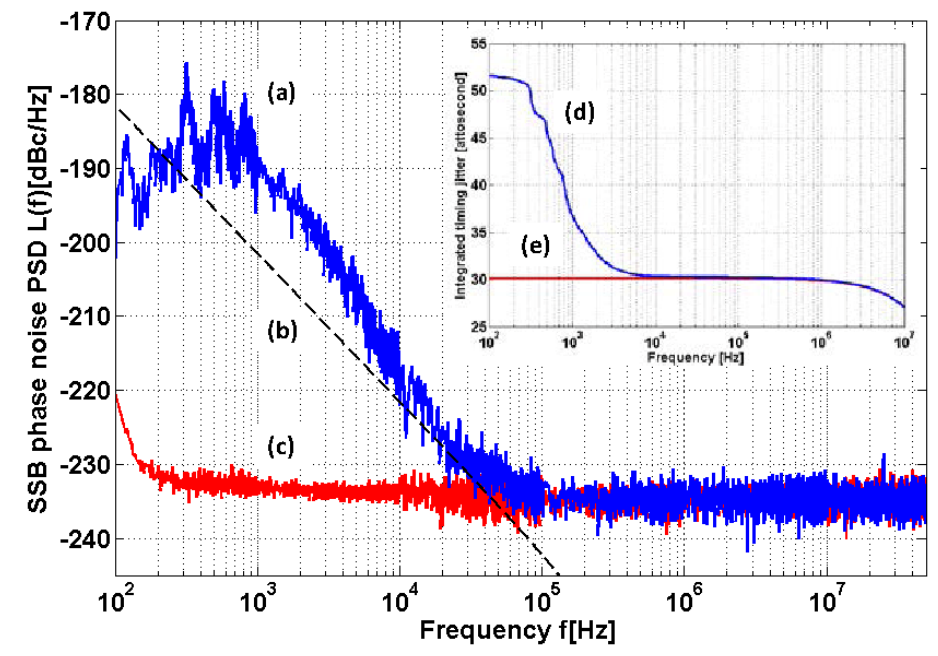

Fig. 6. SSB phase noise PSD of a single laser at 100-MHz carrier. (a) Measured Cr:LiSAF phase noise (b) Quantum-limited estimation, summing up all three noise sources in Fig. 2. (c) Balanced detector's noise floor. Inset: integrated timing jitter from $50 \mathrm{MHz}$ (d) Cr:LiSAF (e) Balanced detector's noise floor.

\section{Intensity noise and timing jitter}

The measured phase noise shown in Fig. 6 strongly deviates from its quantum-limited prediction. This implies the existence of excess technical noise, possibly from the limited CMRR and/or intensity noise coupling to timing jitter. The influence of CMRR can be tested by measuring timing noise at different SFG power levels. Further studies show that varying SFG power only changes measurement sensitivity. This excludes insufficient CMRR from system error.

In order to verify the coupling between intensity noise and timing jitter, the RIN of the pump laser diodes and the mode-locked Cr:LiSAF laser are measured at baseband. This avoids technical difficulties of measuring intensity noise at carrier or harmonic frequencies in the case of Cr:LiSAF [33]. Instead of a trans-impedance amplifier, a $50-\Omega$ resistor is used as front-end. This has the advantage of simplicity and large bandwidth, though precautions must be paid to achieve reliable measurements. On the one hand, the photocurrent $i_{p}$ has to be more than $1 \mathrm{~mA}$, so that shot noise $i_{s}=\left(2 e i_{p}\right)^{1 / 2}$ surpasses Johnson-Nyquist noise $i_{j}=2(k T R)^{1 / 2}$ for $R=50 \Omega$ at $T=300 \mathrm{~K}$. On the other hand, the photocurrent must not exceed the linearity limit of the photodiode. In any case, localized saturation due to overly tight focusing should always be avoided. This suggests that a large-area photodiode should be used in order to achieve high dynamic range.

In this experiment, a $150-\mathrm{MHz}$ Si photodiode (EG\&G FFD040) is reverse biased by a 22$\mathrm{V}$ battery. The laser beam is loosely focused and fills the whole aperture of the built-in iris of the photodetector. The photocurrent is kept between $1 \mathrm{~mA}$ and $2 \mathrm{~mA}$, and terminated by a $50 \Omega$ carbon film resistor. The voltage is then amplified by a home-built AC-coupled lownoise voltage amplifier ( $34.2 \mathrm{~dB}$ gain up to $50 \mathrm{MHz}$ bandwidth) and sent to an oscilloscope or spectrum analyzer. For a given laser, multiple measurements at different photocurrents are compared to ensure no localized saturation or nonlinear response of the photodiode. In addition, the shot-noise limited measurement floor is always calculated as a quick check of photocurrent saturation. As shown in Fig. 7(a), the pump laser diode exhibits $f^{-1}$-like frequency-dependent intensity noise up to $\sim 10 \mathrm{kHz}$, which is typical for Fabry-Perot cavity quantum-well semiconductor lasers due to material and manufacturing defects [34]. If modehopping exists when the laser diode output consists of multiple longitudinal modes, the 
measured RIN shows strong noise peaks, especially at Fourier frequencies beyond $1 \mathrm{MHz}$. The mode-hopping noise can be avoided by operating the laser diode at a combination of forward current and case temperature that results in single longitudinal mode lasing. The mode-locked intensity noise of the Cr:LiSAF laser is shown in Fig. 7(b). At frequencies below $\sim 10 \mathrm{kHz}$, a $f^{-1}$-like noise spectrum is observed, due to the corresponding intensity noise of the pump laser diodes. The relaxation oscillation peak is centered around $100 \mathrm{kHz}$. At high frequencies, the intensity noise rolls off quickly to the shot-noise limited detection floor.

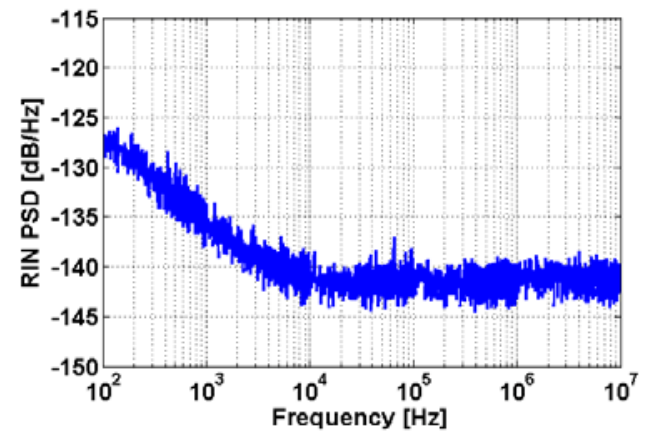

(a)

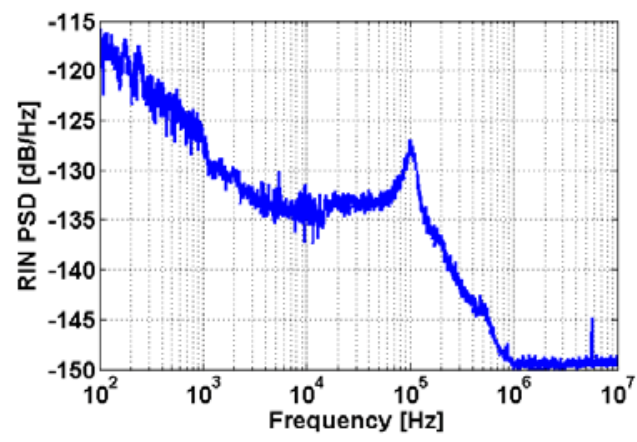

(b)

Fig. 7. RIN of (a) pump laser diode and (b) mode-locked Cr:LiSAF laser.

The transfer function that couples pump RIN and timing jitter is experimentally characterized. The network analyzer in Fig. 8(a) modulates one of the pump laser diodes, and coherently detects the response of the BOC. The measured transfer function is then applied to the pump RIN PSD, which is shown in Fig. 7(a). Such calculated timing jitter due to intensity fluctuations is plotted as the dashed curve in Fig. 8(b). The solid curve in Fig. 8(b) is the same phase noise as shown in Fig. 6(a). For frequencies below $\sim 10 \mathrm{kHz}$, the dashed curve is clipped by the PLL which is closed at a different state than that in the phase noise measurement due to pump modulations induced by the network analyzer; whereas the part beyond $\sim 100 \mathrm{kHz}$ is not real, due to strong filtering of the relaxation oscillation. The rest of the dashed curve closely tracks the measured phase noise PSD. This suggests that the timing jitter of the Cr:LiSAF laser is currently affected by the intensity noise of the diode pump source, in that the pump RIN is transferred to that of the mode-locked laser, which is further converted into timing jitter.

The AM-to-PM coupling occurs either through the self-steepening effect [19] or pulse reshaping upon saturation on the slow saturable absorber [27], or both. These coupling mechanisms are formulated as follows for fundamentally mode-locked lasers. First, the excess SSB phase noise PSD due to the self-steepening effect is $[9,19]$

$$
\mathcal{L}(f)=\frac{1}{2}\left(\frac{f_{\mathrm{rep}}^{2} \varphi_{\mathrm{NL}}}{\pi f \nu}\right)^{2} S_{\mathrm{I}}(f)
$$

where $\varphi_{\mathrm{NL}}$ is the average nonlinear phase per round-trip introduced by the gain medium, $S_{\mathrm{I}}(f)$ intensity noise PSD of the mode-locked laser, and $v$ the center optical frequency. In addition, the AM-to-PM conversion due to the slow saturable absorber is characterized by the phase noise PSD [9]

$$
\mathcal{L}(f)=\frac{1}{2}\left(\frac{f_{\text {rep }}^{2}}{f} \frac{\partial \tilde{t}_{\mathrm{s}}(S)}{\partial S} S\right)^{2} S_{\mathrm{I}}(f)
$$


where $S=w / w_{\text {sat }, \mathrm{A}}$ is the saturation parameter, $w_{\mathrm{sat}, \mathrm{A}}$ the saturation energy of saturable absorber, and $\tilde{t}_{\mathrm{s}}(S)$ the timing shift per round-trip which is thoroughly discussed in [27].

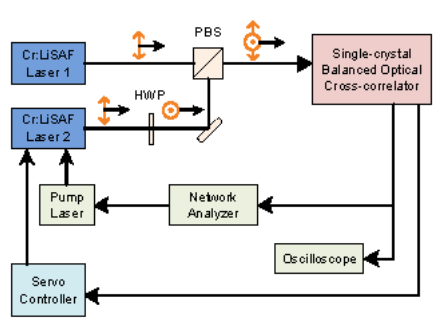

(a)

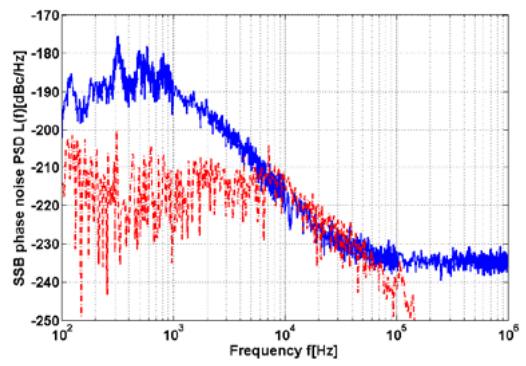

(b)

Fig. 8. RIN noise and timing jitter. (a) Measurement setup. (b) Pump RIN mapped to phase noise (dashed), compared with measured phase noise using BOC (solid, reproduced from curve (a) in Fig. 6).

The saturation parameter is one of the key factors to differentiate the above two mechanisms in terms of coupling strength. It can be identified by comparing intensity noise when the laser runs in continuous-wave and mode-locked regimes, because the saturable and non-saturable loss in mode-locked operation introduces additional cavity loss and therefore alters the laser dynamics [35].

The rate equations for four-level systems give the following coupled differential equations for the dynamics of the intracavity power $P$ and roundtrip amplitude gain $g$.

$$
\begin{aligned}
& T_{\mathrm{R}} \frac{\mathrm{d} P}{\mathrm{~d} t}=2[g-l-q(P)] P \\
& T_{\mathrm{R}} \frac{\mathrm{d} g}{\mathrm{~d} t}=-\frac{g-g_{0}}{\tau_{\mathrm{L}} / T_{\mathrm{R}}}-g \frac{P}{P_{\text {sat } \mathrm{L}}}
\end{aligned}
$$

where $\tau_{\mathrm{L}}$ is the upper-state lifetime of Cr:LiSAF, $l$ the loss, $P_{\text {sat. } \mathrm{L}}$ the gain saturation power, and $q(P)$ the saturable absorber's power-dependent loss. The small signal gain $g_{0}$ is related to pump power $P_{\mathrm{p}}$ by

$$
g_{0}=\tau_{\mathrm{L}} \eta_{\mathrm{L}} \eta_{\mathrm{P}} \frac{P_{\mathrm{P}}}{w_{\text {sat, } \mathrm{L}}}
$$

where $\eta_{\mathrm{L}}$ is the quantum efficiency, $\eta_{\mathrm{p}}$ the pump efficiency and $w_{\text {sat, }}$ the gain saturation energy. Equation (9) explicitly includes pump intensity in the rate equations. Rewriting the intracavity power, gain and pump power in terms of steady-state values, denoted by subscript $s$, and small perturbations, the dynamic equations for intracavity power and gain fluctuations are

$$
\begin{aligned}
& \frac{\mathrm{d} \Delta P}{\mathrm{~d} t}=\frac{2 P_{\mathrm{s}}}{T_{\mathrm{R}}} \Delta g-\frac{2 P_{\mathrm{s}}}{T_{\mathrm{R}}} \frac{\mathrm{d} q(P)}{\mathrm{d} P_{\mathrm{s}}} \Delta P \\
& \frac{\mathrm{d} \Delta g}{\mathrm{~d} t}=-\frac{1}{\tau_{\text {stim }}} \Delta g-\frac{g_{\mathrm{s}}}{w_{\text {sat }, \mathrm{L}}} \Delta P+\frac{\eta_{\mathrm{p}} \eta_{\mathrm{L}}}{w_{\text {sat }, \mathrm{L}}} \Delta P_{\mathrm{P}}
\end{aligned}
$$


where $\tau_{\text {sim }}$ is the stimulated lifetime. By applying the Laplace transform to Eq. (10), the following transfer function describes fluctuations of the mode-locked laser's average power $\widetilde{\Delta P}$ as a result of pump power variation $\widetilde{\Delta P}$

$$
\frac{\widetilde{\Delta P}}{\widetilde{\Delta P}}=\frac{2 \frac{r-1}{T_{\mathrm{R}}} \frac{\eta_{\mathrm{p}} \eta_{\mathrm{L}}}{\tau_{\mathrm{L}}}}{s^{2}+\left(\frac{1}{\tau_{\text {stim }}}+\frac{2 P_{\mathrm{s}}}{T_{\mathrm{R}}} \frac{\mathrm{d} q(P)}{\mathrm{d} P_{\mathrm{s}}}\right) s+\frac{2(r-1)}{T_{\mathrm{R}}} \frac{l+q_{\mathrm{s}}}{\tau_{\mathrm{L}}}+\frac{2 P_{\mathrm{s}}}{T_{\mathrm{R}}} \frac{\mathrm{d} q(P)}{\mathrm{d} P} \frac{1}{\tau_{\text {stim }}}}
$$

where $r=1+P_{\mathrm{s}} / P_{\text {sat, } \mathrm{L}}$ is the pump parameter. In the case of continuous-wave lasing, Eq. (11) is reduced to

$$
\frac{\widetilde{\Delta P}}{\widetilde{\Delta P}}=\frac{2 \frac{r-1}{T_{\mathrm{R}}} \frac{\eta_{\mathrm{p}} \eta_{\mathrm{L}}}{\tau_{\mathrm{L}}}}{s^{2}+\frac{1}{\tau_{\text {stim }}} s+\frac{2(r-1)}{T_{\mathrm{R}}} \frac{l+q_{\mathrm{s}}}{\tau_{\mathrm{L}}}}
$$

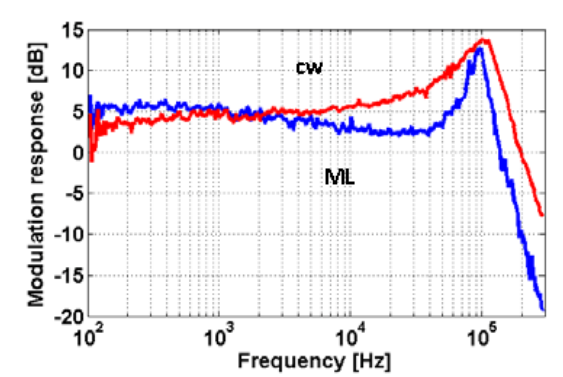

(a)

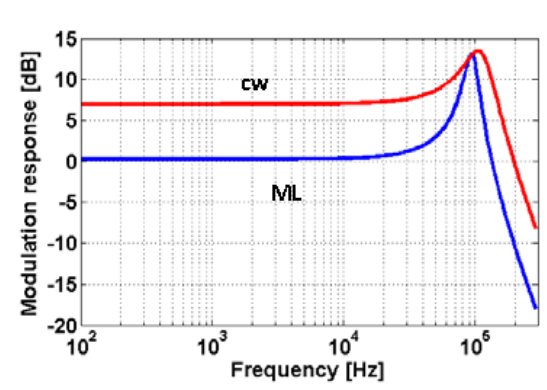

(b)

Fig. 9. Transfer functions (magnitude) from pump RIN to Cr:LiSAF laser RIN. (a) Measured transfer functions. ML: mode-locked, cw: continouse-wave (b) Modeled responses using Eqs. (11) and (12).

The transfer functions described in Eqs. (11) and (12) are experimentally measured using a network analyzer. The pump laser diode driving current is modulated from $100 \mathrm{~Hz}$ to 300 $\mathrm{kHz}$, and the response of both pump laser intensity and Cr:LiSAF laser intensity is coherently detected. In Fig. 9(a), it is clear that when the laser is mode-locked, the saturable absorber's power-dependent $\operatorname{loss} q(P)$, as well as its non-saturable $\operatorname{loss} q_{0}$ included in loss $l$, changes the relaxation oscillation peak's height and width significantly. In order to quantify such a variation, the curves are fitted to results obtained from Eqs. (11) and (12), which are plotted in Fig. 9(b). Although the model is incapable of predicting the full spectral shape, it effectively describes the relaxation oscillation behavior around $100 \mathrm{kHz}$.

To match the measured transfer function to Eq. (11), the parameter

$$
\frac{2 P_{\mathrm{s}}}{T_{\mathrm{R}}} \frac{\mathrm{d} q(P)}{\mathrm{d} P_{\mathrm{s}}}=-0.6 \frac{1}{\tau_{\text {stim }}}
$$

is used. Rewriting of Eq. (13) in terms of the saturation parameter gives

$$
\frac{\mathrm{d} q(S)}{\mathrm{d} S}=-0.6 \frac{2 r P_{\mathrm{s}}}{\tau_{\mathrm{L}}} \frac{w}{S}
$$


In Eq. (14), we have assumed a non-zero $S$, because extremely small values of saturation parameter are not practical to support stable cw-modelocking operation.

The saturable absorber used in these studies has both a fast and a slow component in the temporal response [23]. When timing jitter is concerned, only the slow component is considered, because it results in asymmetric pulse shaping and therefore shifts the pulse center. Assuming that the saturable absorber is slow compared to the pulse duration, it fully recovers within the round-trip time, and the saturable loss is simply

$$
q(S)=\frac{q_{0}}{S}\left(1-e^{-S}\right)
$$

Equations (14) and (15) are sufficient to solve for $S$. In the case of Fig. 9(b), $S=9.7$. In addition, the beam waist on the saturable absorber is calculated to be $45 \mu \mathrm{m}$ using ABCDmatrix analysis, and the saturation flux of the saturable absorber is estimated to be $130 \mu \mathrm{J} / \mathrm{cm}^{2}$. This is a typical value for the saturable absorbers used in our experiments.

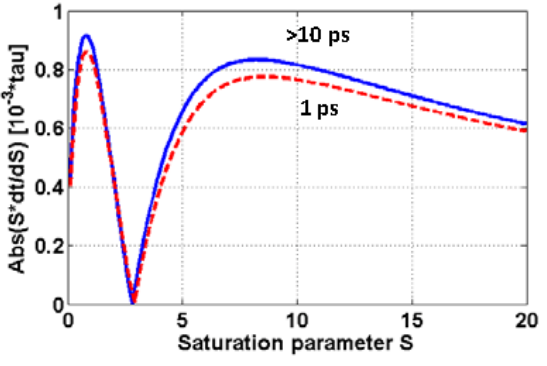

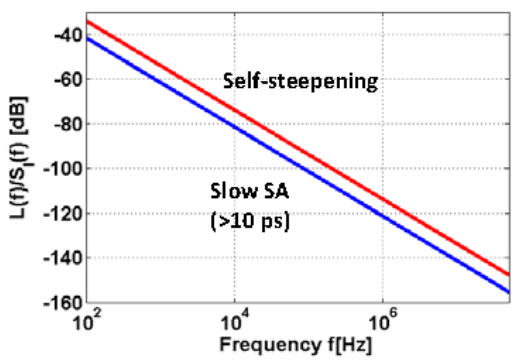

Fig. 10. AM-to-PM conversion factors. (a) Absolute value of $\mathrm{S} * \mathrm{dt} / \mathrm{dS}$ in units of one thousandth pulse width for $1 \mathrm{ps}$ and $>10$ ps recovery time (b) Coupling factors due to selfsteepening and timing shift on slow saturable absorber (SA) with recovery time longer than 10 ps and $\mathrm{S}=8.2$.

The absolute value of parameter $S \cdot\left[\partial \tilde{t}_{\mathrm{s}}(S) / \partial S\right]$ in Eq. (7) and the coupling factors in Eq. (6) and (7) are plotted in Fig. 10. In these plots, 1\% modulation depth is assumed. As shown in Fig. 10(a), the temporal shift of the pulse increases with longer recovery time, but remains virtually constant when the recovery time is sufficiently large (100 times longer than pulse duration). The absolute value of $S \cdot\left[\partial \tilde{t}_{\mathrm{s}}(S) / \partial S\right]$ is maximized at $S=8.2$. The value corresponding to $S=9.7$, where the saturable absorber operates in these studies, is very close to the peak value. In addition, the parameter becomes zero when $S \approx 3$, at which the timing shift due to the saturable absorber vanishes. The coupling strengths due to the self-steepening effect and temporal shift due to the saturable absorber are both plotted in Fig. 10(b). The selfsteepening coupling factor is $7.6 \mathrm{~dB}$ stronger than that of the slow saturable absorber. In other words, the self-steepening effect dominates in the case of our mode-locked Cr:LiSAF lasers.

The magnitude of the temporal shift due to the saturable absorber is sensitive to modulation depth. For a given pulse duration, the peak temporal shift scales linearly with modulation depth, and therefore the coupling factor in Eq. (7) scales as the square of the ratio change. For example, if the modulation depth doubles to $2 \%$, there will be a 6 -dB increase in the AM-to-PM coupling strength due to the saturable absorber. In this case, the effects of selfsteepening and slow saturable absorption become comparable. However, a large modulation depth usually leads to q-switching instabilities. Our experiments with femtosecond Cr:Colquiriite lasers show that saturable absorbers with modulation depths below $1.5 \%$ can start and maintain stable continuous-wave mode-locking, whereas modulation depths larger than $2 \%$ usually result in q-switched mode-locking. This implies that, for femtosecond $\mathrm{Cr}$ :Colquiriite lasers, the contribution of saturable absorption to pulse temporal shift is often 
negligible as long as the laser is stably mode-locked. In the case where AM-to-PM coupling due to the saturable absorber is strong, a relatively small saturation parameter is preferred, because the effect is minimized as $S$ approaches 3 .

\section{Conclusion}

Characterizing attosecond timing jitter of ultrafast solid-state lasers is of great importance. As mode-locked fiber lasers demonstrate sub-100-as timing jitter at quantum limit, the studies on diode-pumped saturable absorber mode-locked Cr:LiSAF lasers show that the upper limit of optical pulse trains timing jitter in such systems is 30 as, integrated from $10 \mathrm{kHz}$ to $50 \mathrm{MHz}$ (Nyquist frequency). The measured timing jitter is mainly limited by the phase detection noise floor and AM-to-PM conversion of pump intensity noise due to the self-steepening effect. By further optimizing the BOC and improving phase detection sensitivity, it would be possible to approach the 13-as timing jitter of KLM Ti:sapphire lasers by the more compact, efficient and directly diode-pumped Cr:LiSAF lasers.

Recently, high-brightness single-mode tapered diode lasers at $675 \mathrm{~nm}$ with $>1 \mathrm{~W}$ output power became available [36]. Such high-power diode lasers have been used to build femtosecond Cr:LiSAF and Cr:LiCAF lasers [37]. Although these diode lasers are currently expensive and their intensity noise remains unreported, the high-power pump source could potentially further reduce footprint and complexity of $\mathrm{Cr}$ :Colquiriite lasers.

The presence of a saturable absorber in femtosecond Cr:LiSAF lasers does not adversely affect timing jitter for a wide range of saturation parameters. The modulation depth of the saturable absorber is usually less than $1 \%$ to ensure stable continuous-wave mode-locking, in which case the AM-to-PM coupling due to pulse temporal shifts of a slow saturable absorber is weak compared to the self-steepening effect, even when the saturable absorber operates at the saturation parameter that maximizes the coupling. For the cases where the peak AM-toPM coupling due to the saturable absorber is strong, it is still possible to properly choose saturation parameters that minimize saturable absorber's influence. In particular, the temporal shift due to the saturable absorber vanishes when $S \approx 3$, which is independent of saturable absorber parameters and operation point, although it is non-trivial to precisely operate the saturable absorber at that point in experiment settings.

The timing jitter study shown here proves that saturable absorber mode-locked ultrafast Cr:LiSAF lasers are promising compact and ultralow jitter sources for optical pulse trains and

microwaves. Future work will be focused on extracting ultralow-noise microwave signals from low jitter ultrafast Cr:LiSAF lasers.

\section{Acknowledgment}

We gratefully acknowledge support from NSF grant ECCS-0900901 and AFOSR grant FA9550-10-1-0063. 